\title{
Bone marrow stromal cell changes in haematological malignancies
}

\author{
S A Dilly, C J Jagger
}

\begin{abstract}
Stromal cell numbers from subjects with no haematological disease and those with acute myeloid leukaemia (AML), chronic granulocytic leukaemia (CGL), acute lymphatic leukaemia (ALL) and non-Hodgkin's lymphoma (NHL) were compared to determine their role in malignancy. Frozen sections of trephine biopsy specimens from iliac crests were stained for endogenous alkaline phosphatase activity, endogenous acid phosphatase activity, and, using immunocytochemical methods, for endothelial cells (anti-factor-VII related antigen) and macrophages and related cells (EBM/11). In granulocytic malignancies, whether acute or chronic, alkaline phosphatase positive reticulum cells (AL-RC) and vascular endothelial cells were generally increased. In lymphoid malignancies, the numbers of AL-RC were generally reduced. Numbers of vascular endothelial cells seemed to be normal in ALL but reduced in foci of NHL. Macrophages are numerous in normal marrow, and their numbers seemed to be normal in granulocytic lesions but were more variable and sometimes reduced in ALL and NHL.
\end{abstract}

Lymphoid malignancies, therefore, have a destructive effect on some stromal elements; granulocytic malignancies are associated with normal or increased numbers of stromal cells. A possible consequence of depleted stromal cells might be slower reconstitution of normal haemopoiesis after treatment. The large numbers in granulocytic malignancies raises the possibility of synergistic stimulation between stromal and neoplastic cells.

Bone marrow stromal cells consist of a mixture of endothelial cells, adipocytes, macrophages and "reticular cells". The use of marrow culture systems, ultrastructural assessment, and immunocytochemical and enzyme histochemical staining has allowed these to become rather better defined in recent years, and two cell types seem to be of particular importance. One is a nonphagocytic cell (AL-RC) (alkaline phosphatase positive reticulum cell) which stains for alkaline phosphatase, is frequently dendritic in shape, and closely associated with granulocytic precursors. The other is a macro- phage commonly associated with erythroid precursors.

AL-RC are stellate cells, frequently adjacent to blood vessels, which have long, thin cytoplasmic extensions partially surrounding vessels and radiating between haemopoietic cells. ${ }^{1}$ Although dendritic in shape, they have neither the staining characteristics nor the antigen-presenting functions of dendritic cells of the lymphoid system. They synthesise reticular fibres important for structural support and also have adhesion molecules, such as fibronectin, which could be important in the anchoring of immature cells. Their cytoplasmic processes partially envelop sinuses and may influence mature haemopoietic cell egress, as the area of sinus wall covered by AL-RC processes is reduced in states of haemopoietic stimulation. They may also produce colony stimulating factors (CSF) as has been found in a cell line believed to be derived from AL-RC. ${ }^{2}$

Macrophage function in the marrow includes phagocytosis of expelled erythroblast nuclei, storage of iron, and probably production of factors stimulatory to erythrocytes, granulocytes, and macrophages. Endothelial cells are involved in migration of haemopoietic cells into the sinuses, are capable of ingesting material from the blood, and may be a source of granulocyte-macrophage CSF. Adipocytes of marrow are very different from adipocytes elsewhere and may be derived from AL-RC. An alternative view is that ALRC and adherent stromal macrophages may be derived from a common progenitor cell. One hypothesis is that the AL-RC produce a lineage non-specific factor for maintaining early stem cells and also macrophage CSF which nurtures marrow derived macrophages. The macrophage itself would produce terminal differentiating hormones. ${ }^{3}$

Interest in the way in which stromal cells control haemopoiesis naturally leads on to their possible role in haematological malignancies. Several oncogene protein products are related to growth factors so, theoretically, oncogenes acting through marrow stromal cells might induce abnormal proliferation of haemopoietic cells, predisposing them to malignant change. This was shown in long term murine marrow cultures infected with a recombinant retrovirus in which avian $v$-src was inserted into amphotropic murine leukaemia virus. It resulted in an increase in macrophages and "large, basophilic adherent cells" five weeks after infection, with decreased numbers of adipocytes. The 
haemopoietic cells were not infected by the virus but were permanently changed so that they had a primitive morphology and did not mature further than progenitor stages. The authors concluded that an oncogene in the stromal cells could facilitate the proliferation of these aberrant, although not frankly leukaemic, cells and this might be a first step in malignant change. ${ }^{4}$

Less is known about stromal cells in intact human marrow. They might be important in maintaining a suitable microenvironment for the growth of malignant haemopoietic cells similar to their interaction with benign haemopoietic cells. Alternatively, they could be damaged or destroyed by the invading malignant cells which might compromise subsequent regrowth of haemopoietic elements after treatment.

\begin{abstract}
Methods
Fifty six bone marrow trephine biopsy specimens were obtained from patients, with their consent, as part of the investigation of their clinical disease. These trephine specimens were divided longitudinally with a razor blade while fresh and then half was formalin fixed for resin embedding and half was mounted on cork in OCT compound and snap-frozen in isopentane and liquid nitrogen.

Paraffin wax sections were cut at $3 \mu \mathrm{m}$ and stained with haematoxylin and eosin or Giemsa, and for chloroacetate esterase and reticulin where appropriate. Frozen sections were used for staining for endogenous alkaline phosphatase, endogenous acid phosphatase, and with an antibody against macrophages, EBM/11 (Dakopatts) ${ }^{5}$ and anti-factor VIII related antigen (Dakopatts). Frozen sections were cut at $6 \mu \mathrm{m}$, mounted on multispot slides, air dried for one to two hours, fixed in acetone for 25 minutes, rinsed in TRIS buffered saline (TBS), pH 7.6, further fixed in paraformaldehyde-periodate-lysine solution at $4^{\circ} \mathrm{C}$ for four minutes, and rinsed in TBS. For demonstra-
\end{abstract}

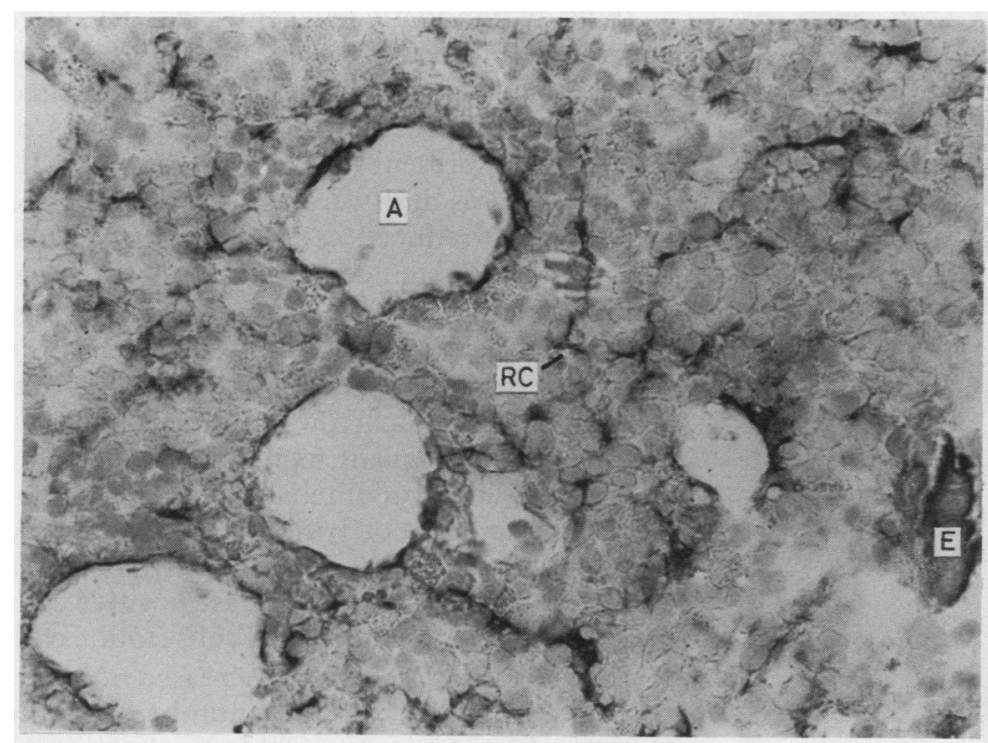

Figure 1 Normal marrow: endogenous alkaline phosphatase. Positive staining is seen on dendritic cells $(R C)$, adipocytes $(A)$, and vascular endothelium $(E)$. tion of endogenous alkaline phosphatase, the sections were then incubated in substrate using the substituted naphthol method with naphthol-ASBI-phosphate and Fast Red TR for 20 minutes. For acid phosphatase, the pararosanolin hydrochloric acid method at $\mathrm{pH} 4.8$ was used. After rinsing in distilled water and counterstaining with Mayer's haematoxylin they were mounted in Apathy's media. For immunocytochemical staining with $\mathrm{EBM} / 11$, primary antibody was applied for one hour, followed by goat anti-mouse secondary antibody $\left(\mathrm{F}(\mathrm{ab})^{2}\right.$ (Sigma)) for 30 minutes, alkaline phosphatase-antialkaline phosphatase (Dako) for 30 minutes, and then substrate as above but including Levamisole to block endogenous activity. For antibody against factor VIII related antigen, alkaline phosphatase labelled secondary antibody was used (goat anti-rabbit whole molecule (Sigma)). All antisera were diluted in 3\% bovine serum albumin in TBS at pH 7.6; secondary antibody had $1 \%$ normal human serum added, and all procedures were at room temperature unless otherwise stated.

The patients studied fell into five categories: normal, acute myeloid leukaemia (AML), chronic granulocytic leukaemia (CGL), acute lymphatic leukaemia (ALL), and non-Hodgkin's lymphoma (NHL). The patients with histologically normal marrow had been biopsied as part of their initial staging for malignant melanoma. They were therefore not entirely clinically normal but had no haematological problems. Although there was histological evidence of active disease in all of the paraffin wax sections, sometimes this was not readily recognisable in the frozen sections and these cases were excluded. This left seven cases of AML, six cases of CGL, six cases of ALL, six cases of NHL and 10 normal controls. In most cases the marrow trephine specimen was obtained at initial presentation before any treatment. In a few cases the patient had relapsed after treatment and the trephine specimens were taken because of clinical suspicion of recurrent disease. All of the cases of nonHodgkin's lymphoma were of follicle centre cell type. No cases of erythroleukaemia were included. The patients with normal marrows had a median age of 51 years, ranging from 34 to 72 years. Six of these patients were men.

\section{Results}

NORMAL MARROWS

Endogenous alkaline phosphatase staining was present in endothelial cells, osteoblasts, some spindle cells, occasional adipocytes and dendritic cells (fig 1). The dendritic cells (AL-RC) were principally within haemopoietic islands and adjacent to small vessels. No concentration of AL-RC was identified in paratrabecular areas where granulopoiesis predominates, although in some marrows AL-RC seemed to be relatively sparse in areas of erythropoiesis compared with sites of granulopoiesis. Areas lacking haemopoietic activity frequently contained rounded or spindle shaped alkaline phosphatase positive cells between the adipocytes. These cells also stained for factor VIII related 
Stromal cell staining in haematological malignancies

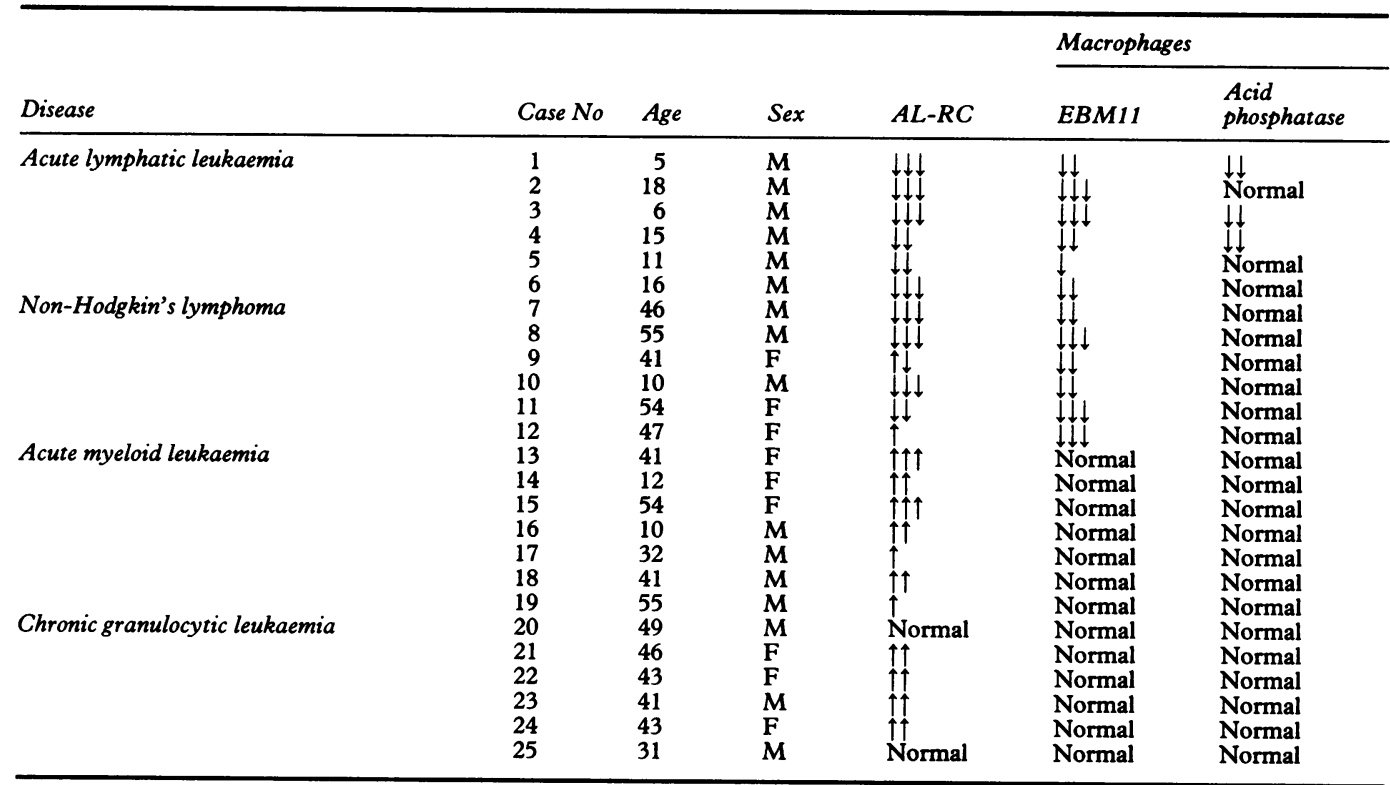

AL-RC = alkaline phosphatase positive reticulum cells; $\uparrow \uparrow \uparrow=$ greatly increased; $\uparrow \uparrow=$ moderately increased; $\uparrow=$ mildly increased; $\uparrow \downarrow=$ variable numbers; $\downarrow=$ mildly decreased; $\downarrow \downarrow=$ moderately decreased; $\downarrow \downarrow \downarrow=$ severely decreased.

antigen and so were small vessels.

$\mathrm{EBM} / 11$ stained the cytoplasm of macrophages, some of which contained phagocytosed iron. The macrophages were numerous, fairly uniformly distributed throughout the marrow, and had no obvious association with any anatomical compartment or type of haemopoiesis. Osteoclasts and megakaryocytes also stained, but no dendritic shaped cells stained. Tartrate sensitive acid phosphatase staining was positive on macrophages and osteoclasts in similar numbers and distribution to EBM/11.

Factor VIII related antigen staining was present on endothelium of both large and small blood vessels and on megakaryocytes. No dendritic shaped cells were stained.

\section{LEUKAEMIC/LYMPHOMATOUS MARROWS}

All seven cases of AML had increased numbers of cells staining for alkaline phosphatase dif-

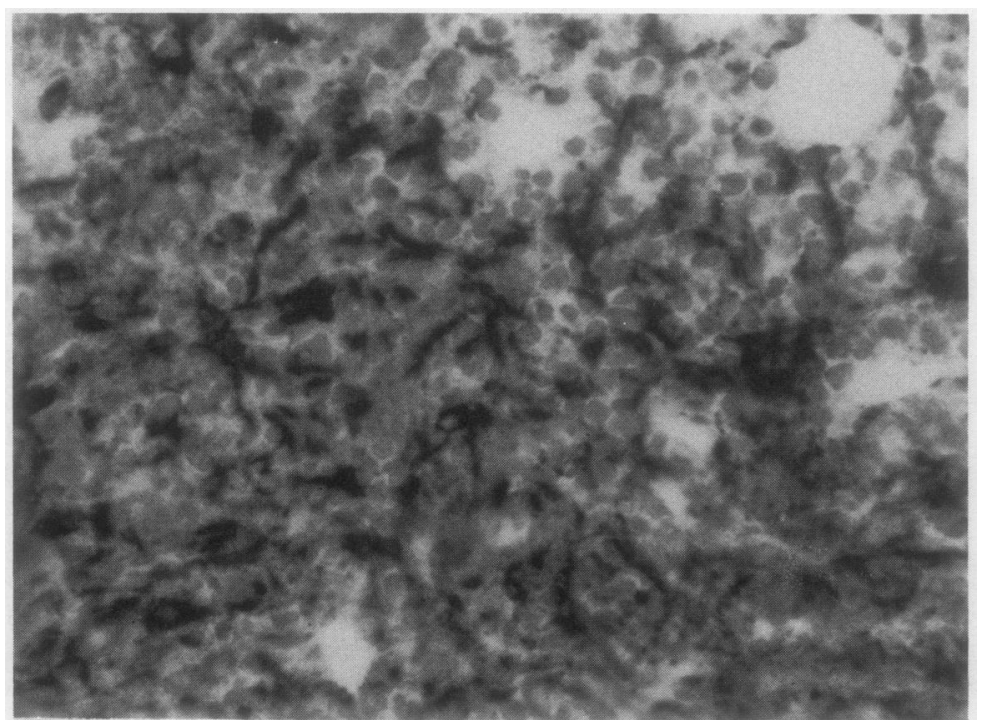

Figure 2 AML: endogenous alkaline phosphatase. A hypercellular marrow with few fat cells but increased positive cells. fusely distributed through affected tissue (table). These were a mixture of dendritic shaped cells (AL-RC) and small and large spindle shaped blood vessel endothelial cells (fig 2). Immature granulocytic cells did not stain and mature granulocyte staining was weak and easily distinguished. The appearances were similar in four of the patients with CGL but in two cases the numbers were normal. Factor VIII related antigen staining was increased due to an increase in vessels and was also present on megakaryocytes. The increase in factor VIII related antigen staining did not account for all of the increase in alkaline phosphatase positive cells and AL-RC were therefore increased. In both AML and CGL macrophages were abundant and definitely not decreased compared with normal, whether stained for acid phosphatase or with EBM/11. Any small increase would be difficult to detect because of the large numbers present in normal marrow.

In acute lymphatic leukaemia, AL-RC were severely reduced in all cases, although vascular endothelial staining for factor VIII related antigen was normal (fig 3). The numbers of macrophages present were far greater than $A L$ RC in all cases and were generally similar or only slightly reduced compared with normal marrow, when stained for acid phosphatase, but more clearly reduced on EBM/11 staining.

In NHL it was important to distinguish the areas affected by lymphomatous cells from adjacent normal marrow. Residual marrow distant from malignant foci had normal numbers of AL-RC, endothelial cells, and macrophages. The areas of lymphoma, which were frequently paratrabecular, generally showed a reduction in alkaline phosphatase positive cells (both AL-RC and endothelial cells (four cases) (fig 4), with one case having increased numbers and one showing variable numbers in the different foci of lymphoma. Macrophage numbers seemed to be reduced when stained for 


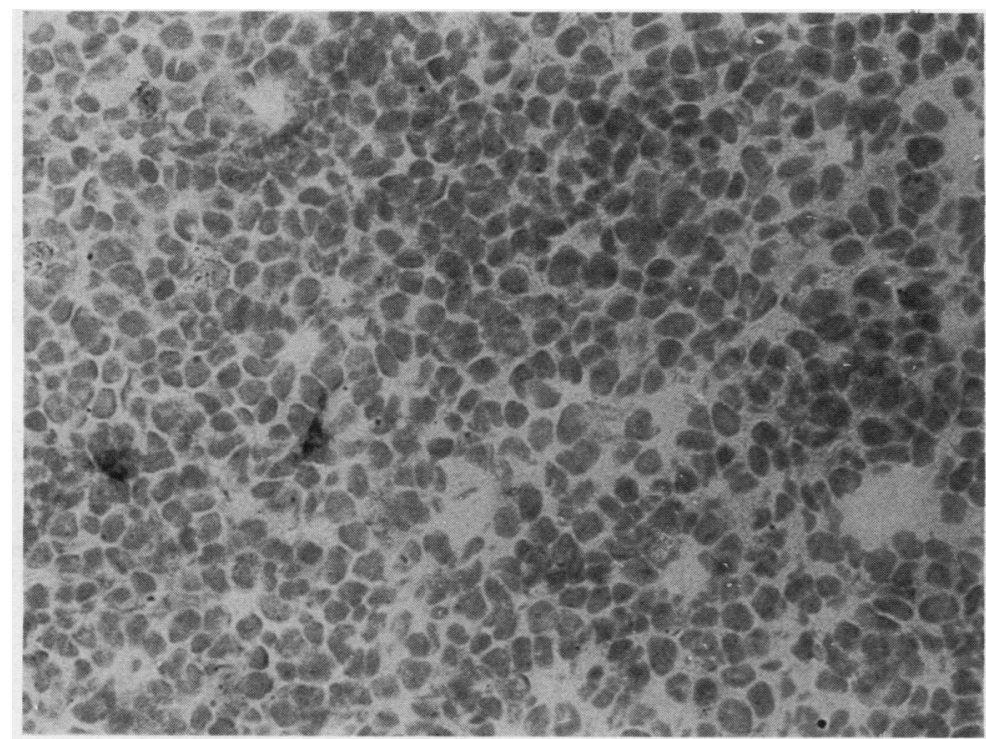

Figure 3 ALL: endogenous alkaline phosphatase. Noticeable reduction in positive cells.

EBM/11 but near normal when assessed for acid phosphatase activity.

No difference was noted between patients biopsied at first presentation and those biopsied during relapse.

\section{Discussion}

This work has shown that bone marrow stromal cells-namely, macrophages, dendritic cells with alkaline phosphatase positivity (ALRC), and endothelial cells with alkaline phosphatase and factor VIII related antigen positivity-show different changes in various haematological malignancies. In granulocytic lesions, whether acute or chronic, the number of AL-RC and endothelial cells generally increase while macrophage numbers remain high as in normal marrows. In contrast, in ALL and NHL, AL-RC are reduced. Vascular endothelial cell staining is normal in ALL and reduced in foci of NHL. Macrophage numbers are near normal or reduced in ALL and NHL,

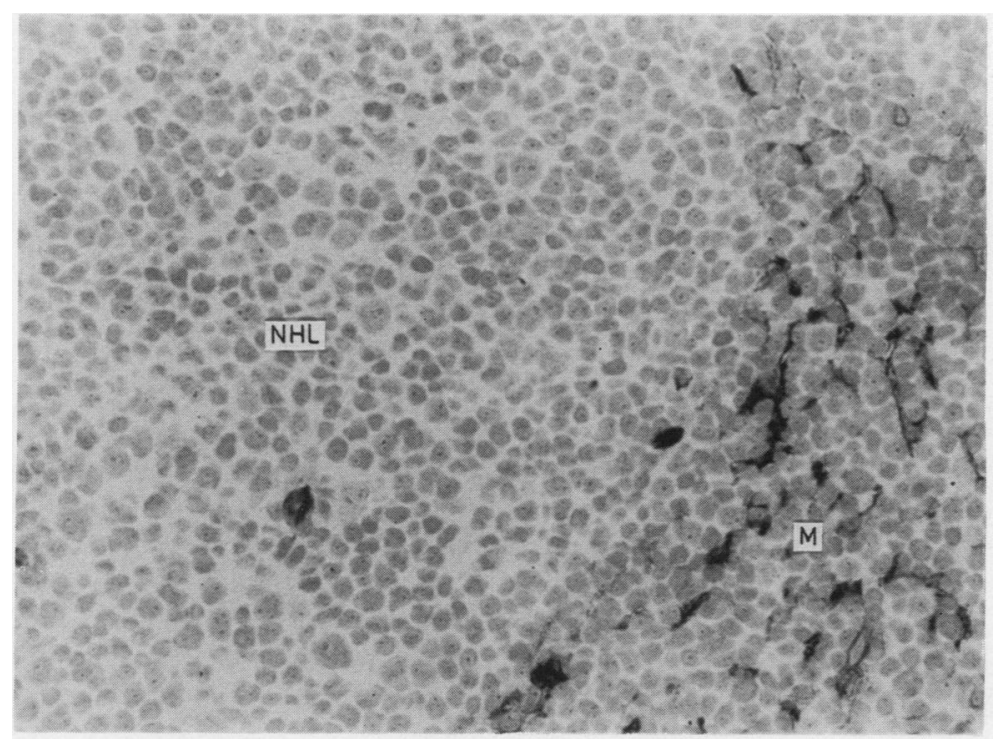

Figure 4 NHL: endogenous alkaline phosphatase. Focus of lymphoma (NHL) with reduction in positive cells contrasts with adjacent marrow (M). depending partly on the method used for their demonstration, but they also show variation among patients.

A problem in assessing AL-RC is the lack of a specific antibody marker. These cells are negative for factor VIII related antigen, macrophage markers, desmin, follicular dendritic cell marker (Dakopatts, DRC-1) and S100 (which stains some antigen presenting dendritic cells). They show, however, strong positivity for vimentin (Authors' unpublished observations). Thus at present, their alkaline phosphatase activity is the best way of identifying them, but it is important to distinguish them from other cells in the marrow with similar activity. These include endothelial cells and some adipocytes. Adipocytes can be readily recognised by their size and fat content and did not pose a problem in this study because the marrows were hypercellular with few adipocytes. Endothelial cells can generally be distinguished because they form tubular structures and do not possess dendritic processes, although an occasional oblique section can cause problems. Because of this, the marrows were stained for factor VIII related antigen so that endogenous alkaline phosphatase activity and factor VIII related antigen distribution could be compared. This showed that vessels were prominent in granulocytic malignancies but did not account for all of the increase in alkaline phosphatase staining.

Numbers of AL-RC were very low in ALL. The patients with ALL were considerably younger than either the control group or other disease groups and so this might have been a consequence of age. This explanation is unlikely, however, as no significant difference was found between young and older patients in a study of stromal cells after bone marrow transplantation, where young patients included three under 8 years old and a further six under 20 years old compared with a mean age of 35 for the older group (authors' unpublished observations). Numbers of AL-RC are also not reduced in fetal and neonatal marrow (Authors' unpublished observations).

Some observations on AL-RC and adipocyte staining for alkaline phosphatase before and after chemotherapy in cases of acute non-lymphoid leukaemia were made by Bianco and colleagues. ${ }^{6}$ They found an abundance of ALRC in marrows devoid of fat cells before treatment, and few or no AL-RC one month after treatment when there were numerous positive early adipocytes. Because of the reciprocal relation between alkaline phosphatase positive adipocytes and AL-RC, and because of previous experimental work, they suggested that one may convert into the other. Our findings of increased AL-RC in granulocytic malignancies could be interpreted as the result of conversion of adipocytes to AL-RC. The same mechanism for the disappearance of adipocytes, however, cannot be proposed for ALL when marrows contained neither adipocytes nor many AL-RC.

AL-RC have several morphological and staining characteristics in common with fibroblasts. Increased numbers could therefore contribute to myelofibrosis in granulocytic 
conditions, although none of the cases we studied had much fibrosis. It has been reported that blood flow is increased through bones with myelofibrosis, possibly with an accompanying increase in number and size of vascular sinuses. This would produce an increase in alkaline phosphatase positive cells of both endothelial and reticular types.

Stromal cells are thought to secrete factors stimulatory to both lymphoid and granulocytic cells, although precise details of their individual capabilities are unknown. ${ }^{37}$ Thus it might not be surprising to find increased numbers of stromal cells if malignant cells retained some responsiveness to promoting factors. Conversely, if no recognition occurred between stromal and malignant cells, then the malignant population could overrun and destroy normal stroma. Cases of NHL frequently showed pronounced reduction of all alkaline phosphatase elements within the focus of tumour and an increase in the adjacent normal marrow, suggesting that the stromal cell network has been pushed out of the way. In ALL the vascular framework was intact, but AL-RC were reduced. Thus lymphoid malignancies have a destructive effect on some stromal elements; granulocytic malignancies do not.

Macrophage numbers in NHL and ALL seemed to be reduced when detected with EBM/11 compared with acid phosphatase. This may be a consequence of macrophage heterogeneity, with not all macrophage populations having identical phenotypes. The interpretation of change in macrophage populations is complex because macrophages may be recruited as an immune response to tumour or a normal reaction to damage. Therefore it would be necessary to perform in situ hybridisation for stimulatory factors to distinguish those primarily involved in promoting haemopoiesis. Similarly, the production of stimulatory factors by AL-RC would be most interesting. A theoretical consequence of depleting cells capable of producing stimulatory factors might be slower reconstitution of normal haemopoiesis after treatment.

We are grateful to Dr J P Sloane of the Royal Marsden Hospital, Sutton, for pathological material, to the Medical Records Department for access to patients' notes, and to Mrs V Emmons for secretarial assistance.

This work was supported by a locally organised research gran funded by the South West Thames Regional Health Authority.

1 Westen H, Bainton DF. Association of alkaline-phosphatase-positive reticulum cells in bone marrow with granulocytic precursors. J Exp Med 1979;150:919-37.

2 Song ZX, Shadduck RK, Innes Jr DJ, Waheed A, Quesenberry PJ. Hematopoietic factor production by a cell line (TC-1) derived from adherent murine marrow cells. Blood 1985;66:273-81

3 Quesenberry PJ, McNiece IK, Robinson BE, et al. Stroma cell regulation of lymphoid and myeloid differentiation. Blood Cells 1987;13:137-46.

4 Dexter TM, Whetton AD, Spooncer E, Heyworth C, Simmons $\mathrm{P}$. The role of stromal cells and growth factors in haemopoiesis and modulation of their effects by the src oncogene. J Cell Sci 1985;(Suppl 3):83-95.

5 Kelly PMA, Bliss E, Morton JA, Burns J, McGee JO Monoclonal antibody EBM/11: high cellular specificity Monoclonal antibody EBM/11: high cellular specificity for human macrophages. J Clin Pathol 1988;41:510-15.
Bianco P, Constantin M, Dearden LC, Bonucci E. Alkaline

6 Bianco P, Constantin M, Dearden LC, Bonucci E. Alkaline phosphatase positive precursors of adipocytes

7 Kincade PW, Witte PL, Landreth KS. Stromal cell and factor dependent B lymphopoiesis in culture. Curr Topics Microbiol Immunol 1987;135:1-21. 